\title{
Do We Need Multiple Biopsies for Assessing Gastric Cancer Risk?
}

\author{
Noriya Uedo
}

Published online: 16 February 2011

(C) Springer Science+Business Media, LLC 2011

It is widely accepted that differentiated-type gastric cancers evolve through a multistep process starting with Helicobacter pylori-associated superficial gastritis, followed by atrophy, intestinal metaplasia (IM), dysplasia, and finally carcinoma [1]. Thus, the identification of such precancerous condition and follow-up of patients in whom they are found could lead to the diagnosis of gastric cancer at early stage and improved patient survival. However, diagnosis of atrophy and IM by conventional white light endoscopy has high inter-observer variability [2] and a poor correlation with histological findings [3]. Consequently, the diagnosis of atrophy or IM is currently based on histology of biopsy specimens from certain anatomic locations of the gastric mucosa, i.e., the updated Sydney system [4]. This system recommends taking at least five biopsy specimens (two from the antrum, two from the corpus and one from the incisura angularis) to grade severity of neutrophils (activity) and lymphocytic infiltration (inflammation), glandular atrophy (atrophy), and intestinal metaplasia. A recent Western publication proposes a staging system to classify gastric cancer risk in clinical practice on the basis of the histological findings of multiple biopsies [5].

The reason for taking biopsies from multiple sites in the stomach is that the grade and distribution of gastritis are different at each site of the stomach in a patient with chronic atrophic gastritis. Mapping studies of biopsy findings suggested that chronic atrophic gastritis develops from the lesser curvature of the lower gastric body and extends

\footnotetext{
N. Uedo $(\square)$

Department of Gastrointestinal Oncologym, Endoscopic Training and Learning Center, Osaka Medical Center for Cancer and Cardiovascular Diseases, 3-3 Nakamichi 1-chome, Higashinari-ku, Osaka 537-8511, Japan

e-mail: uedou-no@mc.pref.osaka.jp
}

upward and laterally in the corpus [6]; therefore, knowledge of the biopsy site is important to interpret histological finding of gastritis in multiple biopsy specimens. When we investigated association between gastric cancer risk and histological grade of gastritis at the each biopsy site in the stomach, the presence of IM in the lesser curvature of the corpus had the strongest association with cancer risk among other findings of gastritis in the other sites [7].

Although gastritis is regarded as a histological entity, many attempts have been made to diagnose the disease macroscopically during esophagogastroduodenoscopy (EGD). Kimura et al. [8] suggested that the endoscopic finding of atrophic mucosa was pale yellowish mucosa with increased mucosal vessel visibility, indicating that it was related to histological finding of atrophy of fundic gland (pseudo-pylorization) by step-wise biopsy across the endoscopic atrophic border. When the atrophic border remained on the lesser curvature of the corpus, the diagnosis was made as closed-type atrophic gastritis (antral predominant gastritis), whereas when the atrophic border no longer exists on the lesser curvature and extends along the anterior and posterior walls of the stomach, the diagnosis was made as open-type atrophic gastritis (pangastritis or corpus predominant gastritis). This endoscopic diagnostic criterion is commonly accepted and practically used for the diagnosis of chronic atrophic gastritis in Japan. Actually, Uemura et al. [9] indicated that extent of mucosal atrophy diagnosed by endoscopy was associated with risk for development of gastric cancer in a large-scale cohort study. In contrast to point evaluation of gastritis by biopsy, endoscopy is advantageous to evaluate the actual extent and distribution of atrophy or IM in the gastric mucosa that is related to gastric carcinogenesis.

Ahn et al. [10] showed that mucosa in patients with open-type atrophic gastritis had more IM and $\mathrm{Cdx} 2$ 
over-expression compared to the mucosa in patients with closed-type atrophic gastritis, implying that this was the reason that they were high risk for developing gastric cancer. In this article, they suggested that a considerable percentage of IM cases were diagnosed solely as open-type atrophic gastritis by endoscopic examination, which might be underestimating the risk for development of gastric cancer. Gastric mucosal atrophy and IM are basically different phenomena but they share a common process in development of Helicobacter pylori-associated chronic atrophic gastritis and are usually present together. Thus, the main finding of their study is understandable.

However, interpretation of the data from the study of Ah et al. [10] requires some discussion of the ability to diagnose IM by conventional white light endoscopy. Although IM in the antrum often appears as slightly elevated whitish patches, IM in the corpus looks flat and can be missed by white light endoscopy. Kaminishi et al. [11] assessed the accuracy of endoscopic findings for diagnosing chronic gastritis and found that ash-colored nodular change was specific (98-99\%) but extremely insensitive (6-12\%) for identifying histological IM [10]. Recently, new endoscopic imaging technology was developed, and its usefulness for diagnosis of gastritis had been introduced. Autofluorescence imaging videoendoscopy (AFI) depicted extent of chronic fundic atrophic gastritis as green mucosa in the gastric corpus and sensitivity and specificity of green mucosa in the gastric corpus in AFI image were 72 and $78 \%$ for atrophy, and 77 and $79 \%$ for IM, respectively [12]. This study revealed that areas with histological atrophy in patients with chronic fundic atrophic gastritis mostly had IM as well. Moreover, usefulness of narrow band imaging (NBI) with magnifying endoscopy, for diagnosis of gastric IM has been reported. Bansal et al. [13] indicated the microsurface structure of ridge/villous pattern in magnifying NBI images for IM had sensitivity of $80 \%$ and specificity of $100 \%$, respectively. We found that a fine light bluish line of light on the epithelial surface or gyri, light blue crest, as a specific finding of IM (sensitivity $89 \%$ and specificity 93\%) [14]. These types of new endoscopic imaging should enable endoscopists to diagnose certain patients with open-type atrophic gastritis as those with metaplastic gastritis (Fig. 1).

Thus, taking all of these observations into consideration, I would like to raise a question from the perspective of a practical endoscopist. Do we need to take many biopsy specimens for assessing gastric cancer risk in patients receiving routine EGD? I think the answer is "no." If we can establish the diagnostic method of endoscopy that corresponds well to histological atrophy or IM, and relate the endoscopic findings to the substantial gastric cancer risk,
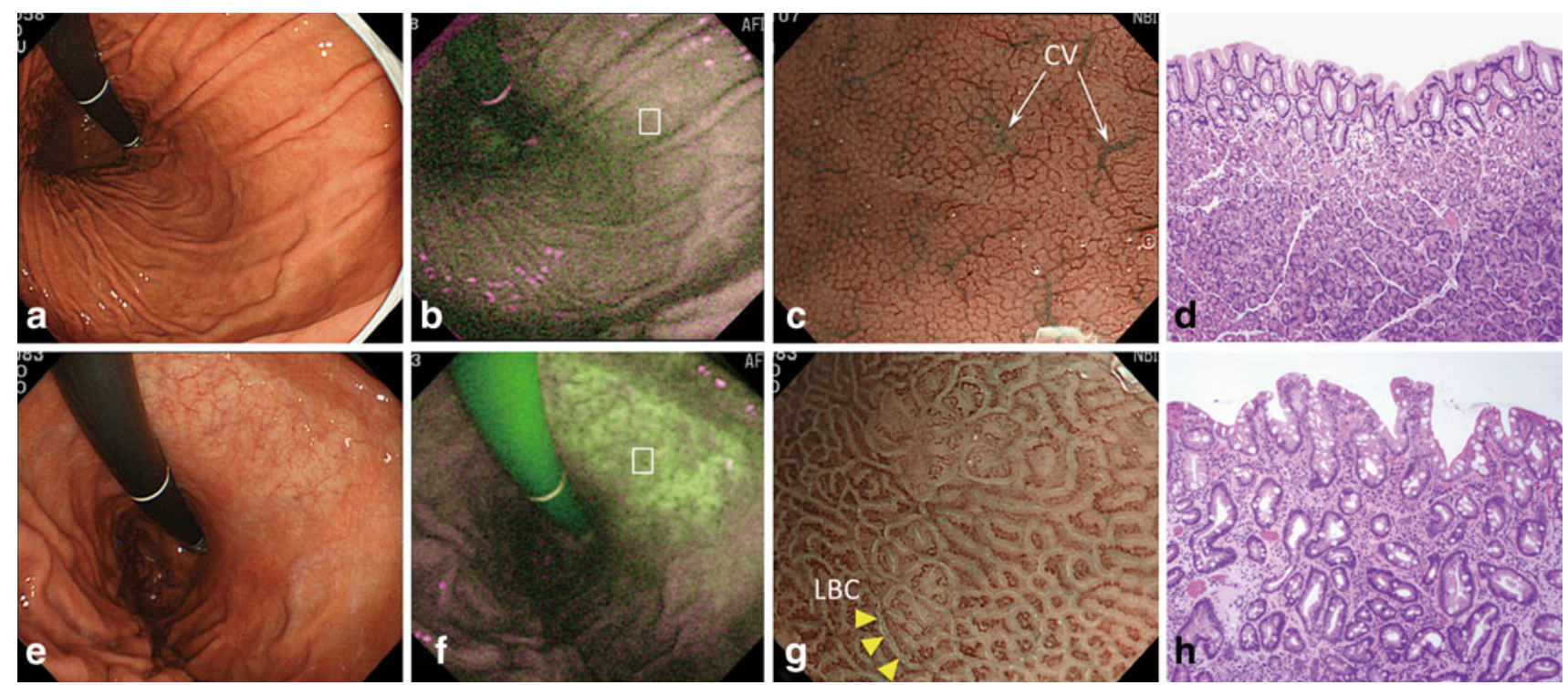

Fig. 1 Helicobacter pylori negative normal corpus mucosa: Mucosa looked homogeneously reddish and the gastric folds were observed circumferentially in the gastric corpus (a). Color of the whole corpus mucosa appeared purple to dark green in AFI image (b). Magnifying image at the lesser curvature of the lower corpus (white box in b) showed small round pits with regularly arranged collecting venule (CV) (c). Biopsy specimen from the corpus lesser curvature had mild inflammatory cell infiltration but no atrophy and no intestinal metaplasia. H\&E, $\times 50$ (d). Closed-type atrophic gastritis mucosa:

Whitish mucosa with increased visibility of vessel was seen in the lesser curvature of the corpus and in gastric folds were absent at that area (e). That area appeared green in AFI image (f). Magnifying NBI image of the green mucosa in the corpus lesser curvature (white box in f) showed ridge/villous surface structure with light blue crest (LBC, yellow arrow head) (g). Biopsy specimen from the corpus lesser curvature had moderate inflammatory cell infiltration, atrophy and intestinal metaplasia. $\mathrm{H} \& \mathrm{E}, \times 50(\mathbf{h})$ 
endoscopy should be enough to assess a risk for development of gastric cancer [15]. Primary screening tests such as H. pylori antibody, serum pepsinogen, family history, etc. may be enough to decide whether or not to perform endoscopy, and we could diagnose the presence and grade of chronic atrophic gastritis and adopt an optimum surveillance program according to stratified risk by endoscopic findings. Histologic findings must increase diagnostic yield of endoscopy to detect gastric cancers at an early stage.

\section{References}

1. Correa P. Chronic gastritis as a cancer precursor. Scand J Gastroenterol. 1984;104:131-136.

2. Laine L, Cohen H, Sloane R, et al. Interobserver agreement and predictive value of endoscopic findings for $H$. pylori and gastritis in normal volunteers. Gastrointest Endosc. 1995;42:420-423.

3. Sauerbruch T, Schreiber MA, Schussler P, et al. Endoscopy in the diagnosis of gastritis: Diagnostic value of endoscopic criteria in relation to histological diagnosis. Endoscopy. 1984;16:101-104.

4. Dixon MF, Genta RM, Yardley JH, et al. Classification and grading of gastritis. The updated Sydney System. International workshop on the histopathology of gastritis, Houston 1994. Am J Surg Pathol. 1996;20:1161-1181.

5. Rugge M, Meggio A, Pennelli G, et al. Gastritis staging in clinical practice: The OLGA staging system. Gut. 2007;56:631-636.
6. Kimura K. Chronological transition of the fundic-pyloric border determined by stepwise biopsy of the lesser and greater curvatures of the stomach. Gastroenterology. 1972;63:584-592.

7. Shiotani A, Iishi H, Uedo N, et al. Histologic and serum risk markers for noncardia early gastric cancer. Int J Cancer. 2005; 115:463-469.

8. Kimura K, Takemoto T. An endoscopic recognition of the atrophy border and its significance in chronic gastritis. Endoscopy. 1969;3:87-97.

9. Uemura N, Okamoto S, Yamamoto S, et al. Helicobacter pylori infection and the development of gastric cancer. $N$ Engl J Med. 2001;345:784-789.

10. Ahn SY, Lee SY, Hong SN, et al. Endoscopic diagnosis of opentype atrophic gastritis is related to the histological diagnosis of intestinal metaplasia and cdx2 expression. Dig Dis Sci. 2011. [Epub ahead of print]. doi:10.1007/s10620-010-1531-8.

11. Kaminishi M, Yamaguchi H, Nomura S, et al. Endoscopic classification of chronic gastritis based on a pilot study by the research society for gastritis. Dig Endosc. 2002;14:138-151.

12. Inoue $\mathrm{T}$, Uedo $\mathrm{N}$, Ishihara $\mathrm{R}$, et al. Autofluorescence imaging videoendoscopy in the diagnosis of chronic atrophic fundal gastritis. J Gastroenterol. 2010;45:45-51.

13. Bansal A, Ulusarac O, Mathur S, et al. Correlation between narrow band imaging and nonneoplastic gastric pathology: A pilot feasibility trial. Gastrointest Endosc. 2008;67:210-216.

14. Uedo $\mathrm{N}$, Ishihara $\mathrm{R}$, Iishi $\mathrm{H}$, et al. A new method of diagnosing gastric intestinal metaplasia: Narrow-band imaging with magnifying endoscopy. Endoscopy. 2006;38:819-824.

15. Hanaoka N, Uedo N, Shiotani A, et al. Autofluorescence imaging for predicting development of metachronous gastric cancer after Helicobacter pylori eradication. J Gastroenterol Hepatol. 2010;25: 1844-1849. 